\title{
Solução da equação diferencial da Gompertz na análise de crescimento de tumores malignos da próstata
}

\author{
Solution of Gompertz's Differential Equantion in the analysis of the growth malig- \\ nant prostate tumors \\ Gentil Fideles Cavalcanti Filho \\ Escola Politécnica de Pernambuco \\ Universidade de Pernambuco \\ 50.720-001 - Recife, Brasil \\ gentilfideles.cavalcantifilho@ya- \\ hoo.com.br \\ Newton Adrião de Aguiar Neto \\ Escola Politécnica de Pernambuco \\ Universidade de Pernambuco \\ 50.720-001 - Recife, Brasil \\ newton_go@hotmail.com \\ Cleomacio Miguel da Silva \\ Escola Politécnica de Pernambuco \\ Universidade de Pernambuco \\ 50.720-001 - Recife, Brasil \\ cleomacio@hotmail.com
}

\begin{abstract}
Resumo Objetivos: este artigo apresenta a solução da Equação de Gompertz com ênfase no crescimento de tumores malignos de próstata utilizando artifícios matemáticos e pesquisas literárias para a determinação dos coeficientes do modelo que melhor se encaixam para as características desse tipo de tumor. Metodologia: foi desenvolvida a solução da Equação de Gompertz e analisada a dependência dessa com cada parâmetro que a compõe, tendo como objetivo, a verificação de qual desses melhor influenciaria nas características do crescimento tumoral de próstata. Com isso, a partir de pesquisas literárias, foi possível determinar cada componente da Equação, chegando ao modelo simplificado do crescimento do tumor da próstata. Resultados: a partir do modelo encontrado, foi possível verificar que o câncer de próstata possui um desenvolvimento lento durante o seu período de desenvolvimento inicial, cerca de 10 anos, sendo praticamente despercebido pelos diagnósticos existentes, porém, após esse tempo, seu desenvolvimento é rápido e consequentemente agressivo, aumentando assim as chances de metástase e complicações na saúde do portador da doença. Conclusão: com este trabalho, foi possível concluir que há uma necessidade do desenvolvimento de novas técnicas para o diagnóstico do câncer de próstata, pois como essa é uma das doenças que mais mata homens por todo o mundo, é grande a importância de prevenir um estado avançado dessa doença.
\end{abstract}

Palavras-Chave: Gompertz, Runge-Kutta, Câncer, Próstata

\begin{abstract}
Objectives: This paper presents the solution of the Gompertz equation with emphasis on the growth of malignant prostate tumors using mathematical tricks and literary research to determine the model coefficients that best fit to the characteristics of this type of tumor. Methodology: was developed solution Gompertz equation and analyzed the dependence that with each parameter within the film, aiming to check which of these best influence the characteristics of tumor growth of prostate. Thus, from literary research, it determined each component of the equation, reaching the simplified model of prostate tumor growth. Results: from the found mod-el, we found that prostate cancer has a slow development during its initial development period, about 10 years, virtually unnoticed by the diagnostic, but after that time, its development is fast and therefore aggressive, thus increasing the chances of metastasis and complications in healthy carrier of the disease. Conclusion: With this work, we concluded that there is a need to develop new techniques for the diagnosis of prostate cancer, for as this is a disease that kills more men around the world, there is a great importance of preventing a state this advanced disease.
\end{abstract}

Keywords: Gompertz, Runge-Kutta, Cancer, Prostate 


\section{Introdução}

Com o desenvolvimento de tecnologias, a qualidade de vida das pessoas melhorou e, entre esses aspectos, está também a saúde em geral, pois com a tecnologia, tornouse possível diagnósticos mais precisos e métodos de tratamentos mais eficazes para várias doenças.

Quando se trata de saúde, uma grande preocupação e assunto para diversos estudos ao redor do mundo é o câncer, que é uma doença caracterizada por uma população de células que cresce e se divide sem respeitar os limites normais, invade e destrói tecidos adjacentes, e pode se espalhar para lugares distantes do corpo, através de um processo chamado metástase.

Câncer é o nome dado a um conjunto de mais de 100 doenças que têm em comum o crescimento desordenado de células, que invadem tecidos e órgãos. Dividindo-se rapidamente, estas células tendem a ser muito agressivas e incontroláveis, determinando a formação de tumores malignos, que podem espalhar-se para outras regiões do corpo [1].

Uma das características malignas de um câncer é sua capacidade de produzir a metástase pois, assim, células cancerígenas se espalham pelo organismo, geram novos tumores no corpo do paciente e, por consequência, a localização de cada um deles se torna uma tarefa difícil e a erradicação cirúrgica pouco eficiente. De fato, metástases são a causa de $90 \%$ das mortes causadas por câncer em humanos [2].

São diversos os tratamentos que a medicina emprega para combater tumores. Eles podem ser físicos, como as radiações, que destroem as células tumorais (particularmente sensíveis às emissões radioativas); cirúrgicos, com que se previne a invasão de outras estruturas, no caso dos tumores malignos; ou quimioterápicos, entre os quais está a administração de substâncias inibidoras da divisão celular (antimitóticos) [3].

Para auxiliar estes tratamentos e também entender melhor o comportamento do desenvolvimento tumoral, faz-se necessário ter uma noção de como um determinado tumor realiza o seu crescimento, para isso, diversas pesquisas tentam simular esse crescimento através de vários modelos matemáticos para fins de utilizar as informações retiradas destes como mais um artifício, tanto para diagnósticos mais recentes, quanto para selecionar em qual estado de desenvolvimento o presente tumor se apresenta, entre outros fatores.

\subsection{Objetivos}

Este artigo tem como objetivo apresentar ao leitor uma forma de analisar o crescimento tumoral do câncer de próstata a partir do modelo da Equação diferencial de Gompertz, mostrando também, os passos adotados para a obtenção te todos os parâmetros que compõe esta equação.

\section{Desenvolvimento}

Em 1825, Gompertz desenvolveu seu modelo para o crescimento populacional de uma determinada espécie qualquer, no qual, mostrou-se bastante eficaz quando comparado com os dados reais do crescimento de uma determinada população. Hoje, seu modelo é bastante utilizado em diversas áreas, em especial, no estudo da análise do crescimento de células tumorais.

De acordo com [4] e utilizando as modificações relacionadas à notação dos parâmetros utilizada por [5] a fim de já introduzir a utilização da Equação de Gompertz para o estudo de um crescimento tumoral, a Equação de Gompertz é descrita de acordo com a Equação 1 logo abaixo.

$$
\frac{d N(t)}{d t}=r N(t) \ln \left(\frac{k}{N(t)}\right)
$$

- $\mathrm{N}(\mathrm{t})$, é a população tumoral de células em um determinado instante $\mathrm{t}$.

- $\quad r$, é a constante de crescimento intrínseco das células, com $r>0$.

- $\mathrm{k}$, é a capacidade de carga do tumor, ou se-ja, é o tamanho máximo que o mesmo pode a-tingir com os nutrientes disponíveis [6]. Aqui, seu valor será tratado como sendo uma constan-te.

A solução da Equação 1 é dada pela Equação 2, como segue:

$$
N(t)=k e^{\ln \left(N_{0} / k\right) e^{-r t}}
$$

A Equação 2 representa o a quantidade de células tumorais em um instante t.

Portanto, $N_{0}$ a quantidade de células tumorais no instante $\mathrm{t}=0$.

Para se obter o valor das constantes da Equação 2 com o objetivo de que esta simule o crescimento tumoral de um câncer de próstata, é necessário que se conheça algumas das características deste tipo de câncer e também do câncer 
em geral para a constante $r$, e supor algumas considerações para k e $N 0$.

Apesar da capacidade de carga, $k$, de um tumor ser intimamente relacionada à quantidade de células tumorais, $N(t)$, no instante $t$, consideraremos que um tumor possui um limite para a quantidade de células que não pode ser ultrapassado e que esse valor é da ordem de 1013 células [7]. Assumindo, portanto, $\mathrm{k}=1013$ células tumorais.

Para a análise do crescimento tumoral de um câncer de próstata, será suposto, assim como foi feito em [8], que o desenvolvimento do tumor é realizado a partir da primeira célula cancerígena presente na próstata, assim, $N_{0}=1$ célula tumoral.

Os sintomas de um câncer de próstata são eventos tardios e, geralmente, ocorrem quando a doença avançou localmente ou produziu metástase. Destacam-se a re-tenção urinária e hematúria [9].

Alguns desses tumores podem crescer de forma rápida, espalhando-se para outros órgãos e podendo levar à morte. A grande maioria, porém, cresce de forma tão lenta (leva cerca de 15 anos para atingir $1 \mathrm{~cm}^{3}$ ) que não chega a dar sinais durante a vida e nem a ameaçar a saúde do homem [1].

Um tumor que possui $1 \mathrm{~cm}^{3}$ pesa em torno de $1 \mathrm{~g}$ e representa cerca de 109 células [10]. Supondo que o tumor desenvolve-se a partir de uma única célula (simu-lação que será realizada neste artigo), quando alcança $1 \mathrm{~cm}^{3}$ já completou 30 duplicações celulares. Mais 10 dup-licações resultaria numa massa de $1 \mathrm{~kg}$, que é o tamanho considerado fatal que um tumor pode alcançar [8]. Essa relação mostra que é possível considerar que a quantidade de células tumorais por volume e também por massa de tumor se mantêm constante.

Partindo dessas informações de tempo de desenvol-vimento de uma certa massa tumoral, e da quantidades de células tumorais que esta terá, é possível encontrar o valor da constante r, pois, partindo dessa relação de pro-porcionalidade, em 15 anos, um tumor terá 109 células. Como este dado está sujeito a erros, é possível considerar um intervalo de segurança de dois anos para mais e para menos, assumindo portanto, que entre 13 a 17 anos, o câncer de próstata terá essa quantidade de células tumo-rias desenvolvidas.

Aplicando essa situação (para $\mathrm{t}=13$ anos, 15 anos e 17 anos), com as constantes antes obtidas ( $\mathrm{k}$ e $N_{0}$ ) na Equação 2, é possível encontrar um intervalo em que a constante $\mathrm{r}$ estará contida nesse problema de contorno.
Para encontrar um único valor de r, uma forma é ponderar os valores do intervalo, dando peso maior ao r correspondente de 15 anos, tirando assim, uma média ponderada, na qual, um resultado possível é r =0,0793 / ano.

Assim, o modelo do crescimento tumoral do câncer de próstata adotado neste artigo será expresso pela Equação 3.

$$
N(t)=10^{13} e^{-29,934 e^{-0,0793 t}}
$$

Uma das principais características de um câncer é a sua capacidade de desenvolver metástase. Para que o tumor possa gerar a metástase, é necessário que ele tenha um volume maior do que $3 \mathrm{~mm}^{3}$ e que tenha sanado as suas necessidades de oxigenação e nutrição [3].

Essas duas condições só serão satisfeitas se o tumor desenvolver a capacidade de promover o crescimento de novos vasos sanguíneos na sua direção. Esses novos vasos proporcionarão a irrigação sanguínea do tumor, sanando as necessidades comentadas e, também, servindo como via de acesso para aquelas células cancerígenas que, ao migrarem, podem gerar novos tumores em outras regiões do organismo. Caso essa vascularização não aconteça, as células do tumor entram em degeneração e necrose [11].

Ao processo de formação desses novos vasos sanguíneos denomina-se angiogênese ou neovascularização; termo que foi utilizado pela primeira vez por Hertig em 1935 para descrever a vascularização na placenta [12].

Nos dias de hoje, esse termo é designado para descrever o crescimento de novos vasos sanguíneos a partir de vasos pré-existentes. Esse processo depende da proliferação de células endoteliais, que são células de revestimento dos vasos sanguíneos [13].

Um tumor não pode crescer acima de 106 células sem desenvolver angiogênese para suprir sangue para o tumor [3].

\section{Resultados}

Dependendo de cada tipo de câncer, as características antes citadas no final da seção anterior, irão desenvolverse em escalas de tempo diferentes, pois cada câncer, em geral, possui sua taxa de crescimento, mesmo quando se possui as mesmas quantidades de células tumorais.

Para o câncer de próstata, essas características irão ocorrer, em média, nos tempos indicados pela tabela 1 . 


\begin{tabular}{|c|c|c|}
\hline Descrição & $\begin{array}{c}\text { Quantidade de } \\
\text { células tumo- } \\
\text { rais, } N(\boldsymbol{t})\end{array}$ & $\begin{array}{c}\text { Tempo, } \boldsymbol{t} \\
\text { (anos) }\end{array}$ \\
\hline Ponto de inflexão & $\begin{array}{c}N(t) \\
=1 \times 10^{13} / e\end{array}$ & 42,862 \\
\hline $\begin{array}{c}\text { Ponto de estabili- } \\
\text { dade }\end{array}$ & $N(t) \rightarrow k$ & $\begin{array}{c}\text { Estimado } \\
\text { através da } \\
\text { visualização } \\
\text { gráfica }\end{array}$ \\
\hline $\begin{array}{c}\text { Tamanho máximo } \\
\text { para o desenvolvi- } \\
\text { mento da angi- } \\
\text { ogênese }\end{array}$ & $N(t)=1 \times 10^{6}$ & 7,806 \\
\hline $\begin{array}{c}\text { Ponto mínimo para } \\
\text { o desenvolvimento } \\
\text { de uma metástase }\end{array}$ & $N(t)=3 \times 10^{6}$ & 8,697 \\
\hline $\begin{array}{c}\text { Massa tumoral de 1 } \\
\text { g }\end{array}$ & $N(t)=1 \times 10^{9}$ & 14,863 \\
\hline $\begin{array}{c}\text { Massa tumoral de 1 } \\
\text { kg }\end{array}$ & $N(t)=1 \times 10^{12}$ & 32,345 \\
\hline
\end{tabular}

Tabela 1: Representação dos principais pontos do desenvolvimento tumoral.

A representação gráfica da Equação 3 está na Figura 1.

Na Figura 1, é possível perceber que, o ponto de estabilidade do tumor de próstata $(N t \rightarrow k)$ ocorre por volta de 130 anos, idade muito alta para a estimativa de vida do homem nos dias atuais, mostrando que a estabilidade do câncer de próstata jamais será atingida através dos mecanismos de crescimento naturais (sem a influência de alguma anormalidade).

Na Figura 2, até aproximadamente 10 anos, a população tumoral permanece bastante pequena, no intervalo de 1-107 células tumorais. Essa quantidade de células corresponde a massas desprezíveis, até a ordem dos miligramas respectivamente, só é possível a sua detecção através de microscopia, mostrando que o diagnóstico através do exame do toque retal (palpável), identifica o tumor em um estado já avançado e que este possui uma idade de aproximadamente 15 anos.

Mesmo o tumor tendo uma massa pequena neste in-tervalo, este, após os valores descritos na Tabela 4 , já desenvolveu a angiogênese e há uma probabilidade de ter desenvolvido metástase, apresentando assim, um fator de risco para a saúde do paciente. A metástase originada do câncer de próstata dará início a tumores em novas partes do corpo, na qual, apresentaram diferentes características do tumor de próstata inicial, podendo ter um crescimento mais rápido e agressivo.

Não é necessário que uma metástase localize-se apenas em um local do corpo, podendo ter as suas células migradas distribuídas em diferentes localizações [7].
Alguns estudos mostra que cerca de $80 \%$ dos homens de 80 anos, que morreram por outros motivos, tinham câncer de próstata e nem eles nem seus médicos desconfiavam [14]. isto pode ser interpretados devido um início tardio do câncer de próstata pois, se por exemplo, este tivesse começado seu desenvolvimento quando um homem tivesse por volta dos 70 anos de idade, este levaria cerca de 10 anos para apresentar um devido crescimento, como foi comentado anteriormente. Da mesma forma, caso o desenvolvimento tivesse ocorrido por volta dos 40 anos de idade, quando este homem apresentasse 70 anos de idade, o tumor já estaria em um estado avançado tendo um crescimento bastante elevado nos 10 anos finais.

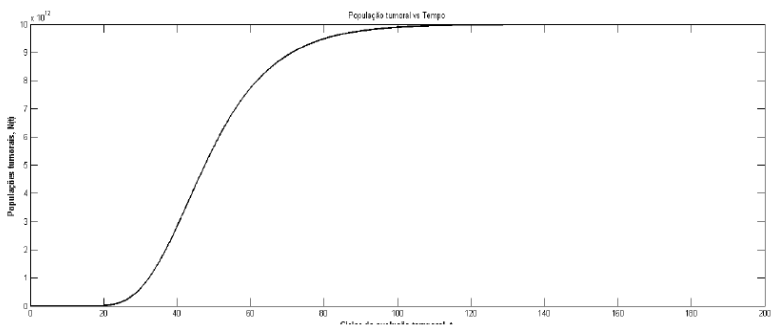

Figura 1: Curva representativa do crescimento tumoral em função do tempo do câncer de próstata

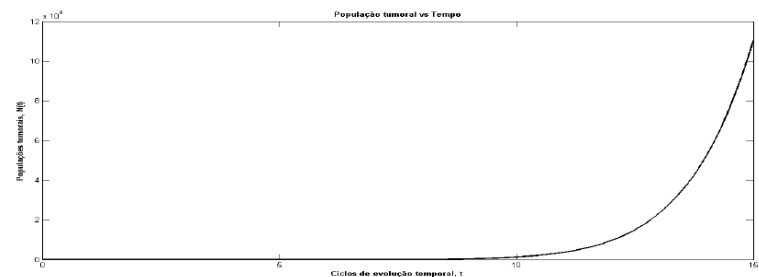

Figura 2: Detalles dos 15 primeiros anos do desenvolvimento do câncer de próstata

\section{Conclusão}

O câncer de próstata é uma doença séria e bastante perigosa que, em muitos casos, não recebe a devida atenção e por consequencia, é desprezada, apresentando assim, um maior risco para a saúde de um paciente.

O modelo da solução da Equação de Gompertz para a análise do crescimento sólido de um câncer de próstata apresentou características baseadas na literatura e que permitem interpretações realistas deste desenvolvimento.

Foi possível concluir que o câncer de próstata possui um desenvolvimento lento durante o seu período de desenvolvimento inicial (cerca de 10 anos), como essa doença geralmente é dada em pessoas de terceira idade que, muitas vezes, sofrem de outros distúrbios, os efeitos do crescimento do tumor é menosprezado ou até mesmo despercebido. Por outro lado, após uma certa massa tumoral, seu desenvolvimento é rápido e consequentemente 
agressivo, aumentando assim as chances de metástase ou até mesmo acarretar outros problemas de saúde.

Esse modelo mostrou também que o exame do toque retal só é capaz de detectar um tumor em um estado já avançado, dificultando assim, o tratamento adequado. Com isso, é importante que a população masculina em geral conheça esta enfermidade, para melhor se adaptarem aos exames de diagnósticos e também, começar este processo em uma idade ideal.

O modelo apresentou também a atual ineficiência dos métodos de diagnósticos, pois, como exemplo, o exame do toque retal identificará um tumor com uma idade aproximada de 15 anos, enquanto este apresentaria características para serem detectados pelo exame de PSA (que detecta tumores com microgramas de massa) em torno dos 7 anos, ou seja, o modelo apresenta o fato de que, os métodos de diagnósticos atuais são incapazes de detectar um tumor quando este apresenta uma pequena quantidade de células tumorais, sendo assim, um desafio para a me-dicina, não só em relação ao câncer de próstata, mas para o câncer como um todo.

\section{Referências}

[1] Instituto Nacional do Câncer (INCA), Disponível em: $\quad<$ http://www2.inca.gov.br/wps/wcm/connect/inc a/portal/home $>$. Acesso em: 01 de julho de 2015 .

[2] S. C. F. Júnior. Modelos de Reação Difusão para o Crescimento de Tumores. Tese de Doutorado, UFMG, Mai 2003.

[3] M. D. Judah Folkman, Tumor angiogenesis: Therapeutic implications; The New England Journal of Medicine. 285:1182-1186, 1971.

[4] W. E. Boyce, R. C. DiPrima. Equações Diferenciais Elementares e Problemas de Valores de Contorno. LTC, Rio de Janeiro/RJ. $8^{\text {a }}$ ed. pág. 49.

[5] J. S. Domingues (2011). Análise do Modelo de Gompertz no crescimento de tumores sólidos e inserção de um fator de tratamento. Bioma-temática IMECC - UNICAMP. MG, Pirapora.

[6] R. K. Sachs, L. R. Hlatky, e, P. Hahnfeldt (2001). Simple ode models of tumor growth and anti- angiogenic or radiation treatment. PER-GAMON Mathematical and Computer Model-ling, 33:1297-1305.
[7] Spencer, S. L., Berrymanb, M. J., Garcia, J. A., e Abbott, D. (2004). An ordinary diferential equation model for the multistep transformation to cancer. Journal of Theoretical Biology, 231:515524.

[8] W. R. Fair, W. D Heston, and C. Cordon-Cardo, Campbell's Urology, ch. (75) An overview of cancer biology, pp. 2259-2282, W. B. Saunders Company, 1997.

[9] D. Seabra, Câncer de próstata - programa educacional, Parte integrante da tese de doutorado, Universidade Federal de São Paulo, UNIFESP-EPM, São Paulo, 2000.

[10] S. Friberg, S. Mattson, 1997. On the growth rates of human malignant tumors: implications for medical decision making. J. Surg. Oncol. 65, 284-297.

[11] J. S. Domingues. Análise do Modelo de Gompertz no crescimento de tumores sólidos e inserção de um fator de tratamento. Biomatemática IMECC - UNICAMP. Dez 2010.

[12] M.A.N.D. Ferreira. Avaliação da angiogênese, in_amação e crescimento tumoral em camun-dongos com deleção gênica dos receptores para o PAF. Tese de doutorado - UFMG, Fev 2006.

[13] A. Fontelonga. Angiogênese. 2009. Disponível em: <http://portal.alert- nline.com>. Acesso em: 10 de julho de 2015 .

[14] A. C. Camargo. Pacientes e Tudo Sobre o Câncer, Próstata. Disponível em: < http://www.accamargo.org.br/tudo-sobre-o-cancer/prostata/32/>. Acesso em: 05 de julho de 2015. 